\title{
The possible origin of facular brightness in the solar atmosphere
}

\author{
R. Kostik ${ }^{1}$ and E. Khomenko ${ }^{2,3,1}$
}

\author{
1 Main Astronomical Observatory, NAS, 03680 Kyiv, Ukraine \\ e-mail: kostik@mao.kiev.ua \\ 2 Instituto de Astrofísica de Canarias, 38205 La Laguna, Tenerife, Spain \\ 3 Departamento de Astrofísica, Universidad de La Laguna, 38205 La Laguna, Tenerife, Spain
}

Received 22 September 2015 / Accepted 21 February 2016

\begin{abstract}
This paper studies the dependence of the Ca II H line core brightness on the strength and inclination of the photospheric magnetic field, and on the parameters of convective and wave motions in a facular region at the center of the solar disc. We use three simultaneous data sets that were obtained at the German Vacuum Tower Telescope (Observatorio del Teide, Tenerife): (1) spectra of Ba II $4554 \AA$ A line, registered with the instrument TESOS to measure the variations of intensity and velocity through the photosphere up to the temperature minimum; (2) spectropolarimetric data in Fe I $1.56 \mu \mathrm{m}$ lines (registered with the instrument TIP II) to measure photospheric magnetic fields; (3) filtergrams in Ca II H that give information about brightness fluctuations in the chromosphere. The results show that the $\mathrm{Ca}$ II $\mathrm{H}$ brightness in the facula strongly depends on the power of waves with periods in the 5-min range, which propagate upwards, and also on the phase shift between velocity oscillations at the bottom photosphere and around the temperature minimum height that is measured from Ba II line. The Ca II H brightness is maximum at locations where the phase shift between temperature and velocity oscillations lies within $0^{\circ}-100^{\circ}$. There is an indirect influence of convective motions on the Ca II $\mathrm{H}$ brightness. The higher the amplitude of convective velocities is and the greater the height is where they change their direction of motion, the brighter the facula. In summary, our results lead to conclusions that facular regions appear bright not only because of the Wilson depression in magnetic structures, but also owing to real heating.
\end{abstract}

Key words. Sun: magnetic fields - Sun: oscillations - Sun: photosphere - Sun: chromosphere

\section{Introduction}

High spatial resolution observations reveal that facular regions break into clusters of bright points, small pores, and facular granular cells (Dunn \& Zirker 1973; Title et al. 1992; Berger et al. 2004; Lites et al. 2004; Narayan \& Scharmer 2010; Kobel et al. 2011; Viticchié et al. 2011). It is thought that these features appear as a consequence of the presence of small-scale magnetic elements (or flux tubes) of the size of hundreds of kilometers and magnetic field strength of the order of 1-2 kG (Stenflo 1973; Solanki 1993). According to numerous theoretical and empirical models (Spruit 1976; Knoelker et al. 1988; Grossmann-Doerth et al. 1994; Topka et al. 1997; Okunev \& Kneer 2005; Steiner 2005), the walls of these tubes are hot and the temperature at the bottom depends on the diameter of the tube. The bottom is cold if the diameter $d$ is greater than $300 \mathrm{~km}$, and it is hot if $d<300 \mathrm{~km}$. Because of the magnetic pressure, the temperature in a thick tube is lower than in the surrounding atmosphere at the same geometrical height. Therefore the bottom of the tube becomes dark in observations. But if the tube is sufficiently narrow, it can be heated by horizontal radiative transfer and becomes visibly bright. The contrast of the tube depends not only on its diameter, but also on the strength of the magnetic field of the tube, such that the larger the field is, the darker the bottom of the tube becomes. This latter dependence makes it possible to test theoretical models by means of observations.

Indeed, observations of the solar disc center given in Frazier (1971), Title et al. (1992), Topka et al. (1992, 1997), Montagne et al. (1996), Kobel et al. (2011), Kostyk (2013) show that the absolute value of continuum contrast of facular regions (without considering separately granules and intergranular lanes) depends on the magnetic field, though the observational findings are somewhat contradictory. Frazier (1971) shows that the continuum contrast of the facula at the solar disc center and the magnetogram signal are correlated in a complicated manner. Below $200 \mathrm{G}$ the contrast increases with the magnetograph signal, while after this value the continuum becomes monotonically darker until the pore and sunspot signals are reached. The Ca II K emission also increases till about $500 \mathrm{G}$. Title et al. (1992) find that the line center brightness of the Ni I $6768 \AA$ line is enhanced in the plage, compared to the quiet area, with a linear dependence between the magnetogram and the brightness, till about $600 \mathrm{G}$, and is decreased afterwards owing to the presence of pores. The continuum contrast is unaffected by the plage magnetic flux till about the same strength and then decreases. Topka et al. (1992) presented evidence that the continuum contrast at $5000 \AA$ of the facula that is close to the disc center is about $3 \%$ less, which is contrary to many earlier observations. Outside of the disc center, the facular becomes brighter in continuum after about $20^{\circ}$. In their latter work, Topka et al. (1997) showed the decrease of the continuum contrast with the strength of the magnetogram signal at the disc center, and explain it in terms of the model of a flux tube with hot walls and a cool floor. In the later work by Montagne et al. (1996) it was shown that the continuum brightness increase is associated with the presence of small-scale magnetic elements in intergranular lanes, leading to an increase of brightness from 0 to $400 \mathrm{G}$, and a decrease afterwards that is associated with the presence of larger structures. The authors used spectra of the Fe I 6301 and $6302 \AA$ lines and 

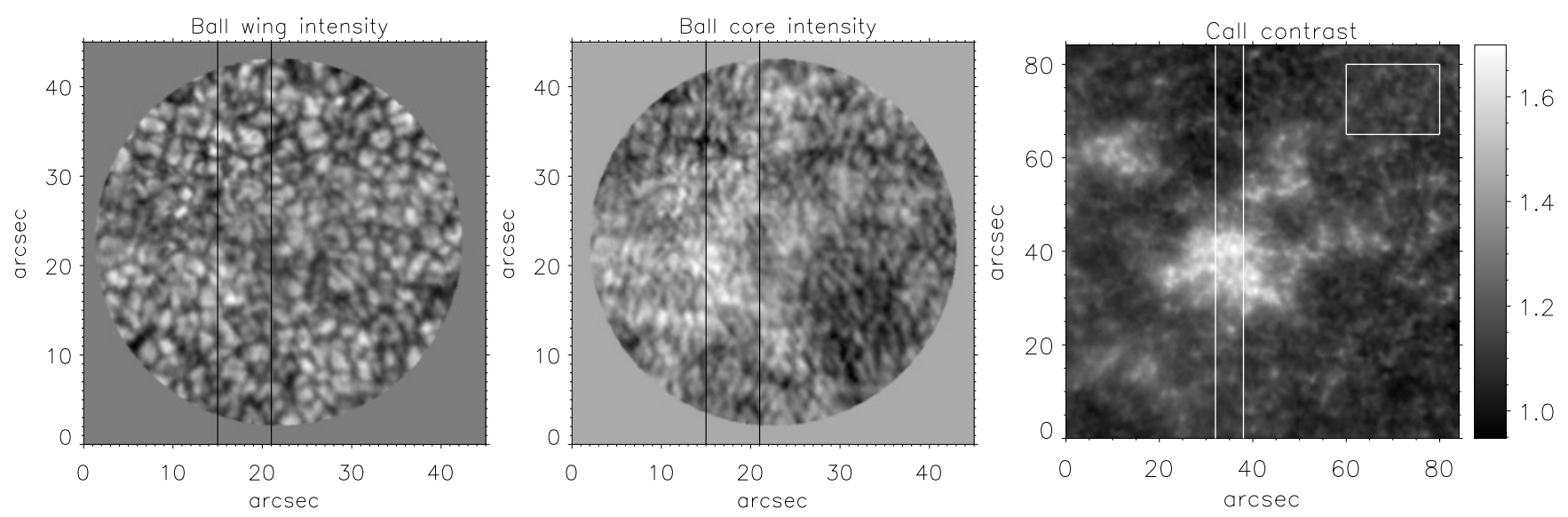

Fig. 1. Complete field of view as detected in Ba II line wing (left), line core (center) and a Ca II H contrast. The vertical lines indicate the area scanned by TIP and used for the scientific analysis in this work. The rectangle area in the right panel indicates the quiet area used as a reference for the definition of Ca II H contrast.

the entire profile was used to calculate the dependencies, which should give more precise results than in Title et al. (1992) and Topka et al. (1992), where the combination of non-simultaneous observations in the two wings of a spectral line was used. In a newer observations by Hinode, with much higher spatial resolution and when the magnetic field strength (not flux) was obtained using inversions, Kobel et al. (2011) find that the relationship between contrast and apparent longitudinal field strength exhibits a peak at around $700 \mathrm{G}$, both for the quiet Sun network and active region plage, while earlier studies only found a monotonic decrease in active regions. The contrast possibly depends on the size of magnetic elements and not on their strength (the intrinsic strength is more or less constant in the $\mathrm{kG}$ range). Kostik \& Khomenko (2012) claim that the observed dependence between brightness and contrast is a consequence of the different distributions of magnetic field strength in granules and intergranular lanes. The histogram of the magnetic field in the facula has two maxima, one at the hecto-G and one at the $\mathrm{kG}$ value. The first maximum has to do with granules, where the field is generally weaker, while the second one is related to intergranular lanes. If one considers the dependence between the contrast and the magnetic field strength, selecting only intergranular lanes, then it turns out that the contrast remains practically unchanged with the magnetic field increasing from 400 to 1600 G, see Fig. 4 in Kostik \& Khomenko (2012). Given the above, the observational dependence between the brightness and other quantities related to granulation needs further studies to clarify if solar faculae are indeed conglomerates of magnetic flux tubes.

The purpose of this work is to deepen the study of the contrast of facular regions at the solar disc center, as observed at the core of Ca II H $3968 \AA$ Aline. Below, we provide the dependences between the Ca II H contrast and the strength and inclination of the magnetic field, as well as various other parameters of solar granular and wave motions.

\section{Observations and data reduction}

Following our previous papers in this series (Kostik \& Khomenko 2012; 2013), we used observations performed at the German Vacuum Tower Telescope (VTT), located at the Observatorio del Teide in Izaña, Tenerife. The dataset was taken on 13 November 2007. Three wavelength regions were observed simultaneously: Fe I $\lambda 15643-15658 \AA$ using TIP-II (Collados et al. 2007), Ba II $\lambda 4554 \AA$ using TESOS
(Tritschler et al. 2002), and Ca II H $\lambda 3968 \AA$ A using a broad-band filter at the VTT. A facular area close to the solar disc center at S05E04 was selected using filtergrams in Ca II H line. Figure 1 gives an overview of the observed area in TESOS (left and middle images) and a filtergram in Ca II H. The vertical lines mark the area that was scanned by TIP. This figure enables us to appreciate the quality of the observational material. It shows that no strong activity was present in the observed area. The granulation appears almost undisturbed, no pores are present. The line core images in Ba II (middle panel) show suspicious brightness in the same locations as in the Ca II H filtergram that correspond to the facular area under study. The data treatment is described in detail in Kostik \& Khomenko (2012, 2013). In the current work, we study temporal variations in an area whose size is $5 . \prime 5 \times 18^{\prime \prime} .5$ during $34 \mathrm{~min}$ and $41 \mathrm{~s}$ of observations. The width of the spectrograph slit was of 0 '”35. Our dataset contains:

- five TIP scan of Stokes spectra of Fe I lines at $1.56 \mu \mathrm{m}$, repeated every $6 \mathrm{~min} 50 \mathrm{~s}$ with a pixel size of $0^{\prime \prime}$. 185 and a spectral sampling of $14.73 \mathrm{m \AA} / \mathrm{px}$;

- time series of Ba II $4554 \AA$ monochromatic images with a temporal resolution of $25.6 \mathrm{~s}$, and a pixel size of 0 '.089, tuned along the spectral line with spectral sampling of $16 \mathrm{~m} \AA / \mathrm{px}$ between successive images;

- time series of Ca II H filtergrams with a temporal cadence of $4.93 \mathrm{~s}$, and a pixel size of 0 '.123.

The seeing-limited angular resolution at the time of observations was no more than $00^{\prime \prime 5}-1^{\prime \prime}$ in the blue range of the spectrum at $4500 \AA$, and around $2^{\prime \prime}$ in the infrared.

We obtained maps of the magnetic field strength and inclination by inverting the Stokes parameters of the Fe I 15648 and $15652 \AA$ lines using the SIR inversion code (Ruiz Cobo \& del Toro Iniesta 1992), see the details in Kostik \& Khomenko (2013). Both the field strength and the inclination were assumed to be constant with height. The intensity and velocity oscillations were found using the $\lambda$-meter technique (Stebbins \& Goode 1987) at 14 levels along the Ba II line profile (i.e. 14 heights in the photosphere, see Shchukina et al. 2009). These are defined as follows (Kostik \& Khomenko 2012):

$\delta I(t, x, W)=I(t, x, W)-\bar{I}(W)$

$\delta V_{\mathrm{r}}(t, x, W)=V_{\mathrm{r}}(t, x, W)-\bar{V}_{\mathrm{r}}(W)$

$\delta V_{\mathrm{b}}(t, x, W)=V_{\mathrm{b}}(t, x, W)-\bar{V}_{\mathrm{b}}(W)$ 
where $\delta V_{\mathrm{r}}$ and $\delta V_{\mathrm{b}}$ are red and blue wing velocities. In the equations above, $W$ means one of 14 reference widths along the line profile, $x$ is spatial position, and $t$ is time. The average intensity levels, $\bar{I}(W)$, and blue and red wing reference positions $\bar{V}_{\mathrm{r}}(W)$ and $\bar{V}_{\mathrm{b}}(W)$ were obtained from the spatially and temporally averaged Ba II profile. The final velocity fluctuations are obtained from red and blue velocities as usual:

$\delta V(t, x, W)=\left(\delta V_{\mathrm{r}}(t, x, W)+\delta V_{\mathrm{b}}(t, x, W)\right) / 2$.

The convective and oscillatory components of the velocity and intensity variations were split using the $k-\omega$ diagram (Khomenko et al. 2001; Kostik et al. 2009).

The Ca II H contrast was calculated with respect to its average value of the quiet area of the same set of observations, $\bar{I}_{\mathrm{Ca}}$, see the rectangular area indicated in the right panel of Fig. 1:

$\delta C(t, x)=I_{\mathrm{Ca}}(t, x) / \bar{I}_{\mathrm{Ca}}-1$,

where $I_{\mathrm{Ca}}(t, x)$ is the intensity in the $\mathrm{Ca}$ II $\mathrm{H}$ filtergram at a given point of a scan. have

Therefore, at each observed pixel of the $5^{\prime \prime} .5 \times 18^{\prime \prime} .5$ area, we

- The strength and inclination of the magnetic field at the deep photosphere;

- Convective and oscillatory variations of the velocity and intensity that are measured along the photosphere (from 0 to $650 \mathrm{~km}$ ) from the Ba II profile;

- Chromospheric intensity variations from the core of $\mathrm{Ca}$ II $\mathrm{H}$ line at a height of about $1000 \mathrm{~km}$.

Figure 2 gives an overview of the above variables in the observed field of view. The comparison between the first two maps from the upper row of this figure with the magnetic field map shows that, at locations with stronger magnetic field, the $\mathrm{Ca}$ II $\mathrm{H}$ contrast is enhanced. The amplitude of the convective velocity also shows larger co-spatial values in the areas with larger $\mathrm{Ca}$ II $\mathrm{H}$ contrast, however its distribution is less homogeneous and more patchy. The power of oscillations of both velocity and $\mathrm{Ca}$ II $\mathrm{H}$ intensity is large at the middle of the observed area, coinciding with the center of the magnetic area. The patch of enhanced oscillation power is smaller than the area of enhanced $\mathrm{Ca}$ II $\mathrm{H}$ contrast. The comparison between the maps of inclination and magnetic field strength reveals that, in the areas where the magnetic field is large (red area), the inclination varies between $0^{\circ}$ and $30^{\circ}$. Outside of this patch the magnetic signal is weaker and we cannot reliably measure the magnetic field inclination. In the analysis below, we only use the area where the inclination does not exceed $40^{\circ}$. The correlations between all the above quantities are discussed below and confirm the visual impression.

We note that the location of the observed region close to the disc center at S05E04 provides that the measured line-of-sight velocity corresponds to the vertical velocity. Therefore the velocity was suitably strong and could be measured reliably. The inclination angles derived from inversions are good proxies for those with respect to the normal to the solar surface.

\section{Results of observations}

Throughout the paper, we use a statistical approach and search for correlations between different quantities. We use either onedimensional dependences, or bi-dimensional ones. Figures 3-5 show the dependence between the $\mathrm{Ca}$ II $\mathrm{H}$ contrast and various
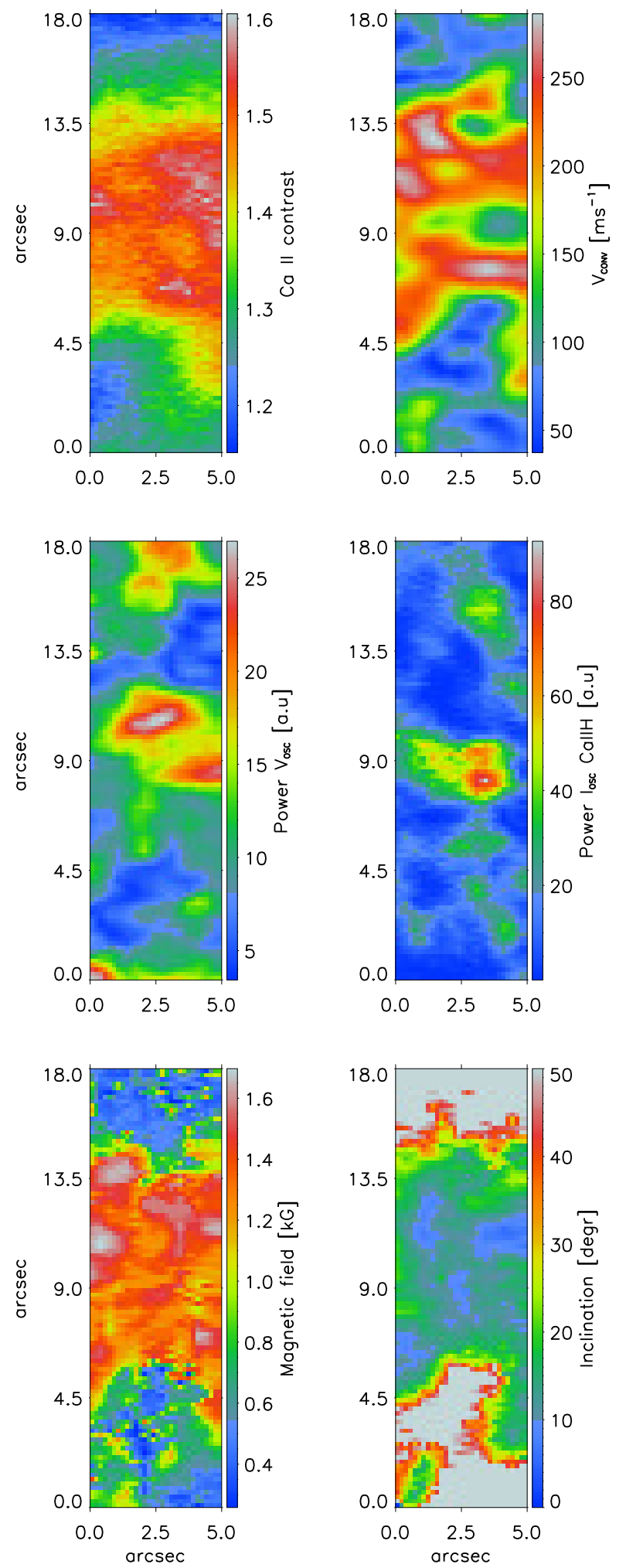

Fig. 2. Upper row: maps of Ca II H contrast (left) and time-average amplitude of the convective velocity at the bottom photosphere (right). Middle row: power of oscillatory velocity component in the upper photosphere (left) and power of oscillatory component of Ca II H intensity (right). Bottom row: map of the magnetic field strength (left) and inclination with respect to the normal to the surface (right) in the observed field of view obtained after inversions of Fe I lines.

parameters of convective and wave motions, and the magnetic field. In the case of one-dimensional dependencies, each point 


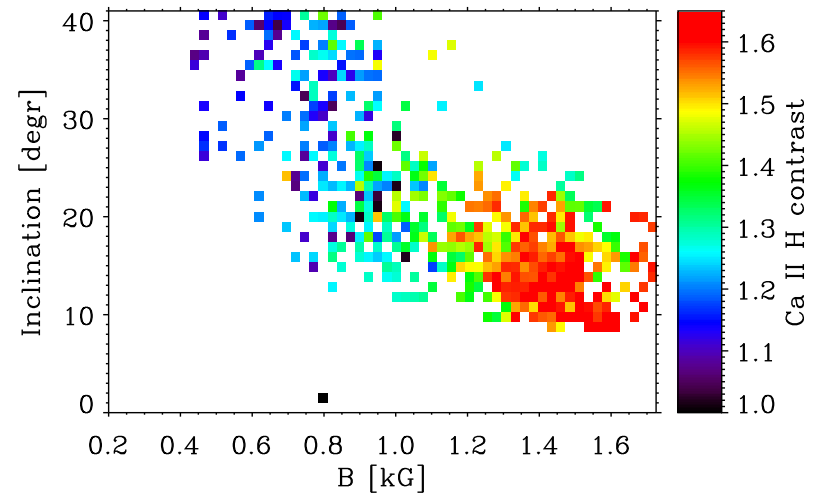

Fig. 3. Bi-dimensional representation of the $\mathrm{Ca}$ II $\mathrm{H}$ contrast as a function of photospheric magnetic field strength and inclination. Each color rectangle in the scatter plot indicates the mean value of the contrast for a given range of field strengths and inclinations. The white areas are the locations where a particular combination of inclination and field strength is not present in our data.

represents the average of $\mathrm{Ca}$ II $\mathrm{H}$ contrast in a bin with an equal number of data points in the abscissa axis. The value of $\sigma$ shown in each panel is calculated as a average standard deviation between the individual and the average values in each bin, and then averaged for all bins. In the case of bi-dimensional plots, the $\mathrm{Ca}$ II $\mathrm{H}$ contrast is averaged for the bins defined jointly for two independent quantities. The latter representation enables us to study dependencies between three parameters simultaneously.

Figure 3 gives a bi-dimensional dependence between the contrast in $\mathrm{Ca}$ II $\mathrm{H}$ and the photospheric magnetic field strength and inclination. We find a clear trend of increasing the contrast from 1.3 to 1.6 with magnetic field strength increasing from 350 to $1400 \mathrm{G}$, with a slight plateau leading to a slightly decreasing contrast around $1650 \mathrm{G}$. The dependence of the contrast on inclination is more monotonic: the contrast decreases from 1.65 to 1.3 with the inclination increasing from $5^{\circ}$ to $40^{\circ}$. As can be seen in the figure, these two trends are not independent. Only a particular combination of the field strengths and inclinations is found in our data, in a way that stronger fields are more vertical. At the same time, for those stronger and vertical fields, the values of $\mathrm{Ca}$ II $\mathrm{H}$ contrast exceed those for weaker and more horizontal fields, as is indicated by the color scheme of the Fig. 3. This trend is also seen in the maps in Fig. 2.

The contrast of the facula as a function of the parameters of convective motions is shown in Fig. 4. We obtain almost a linear dependence between the $\mathrm{Ca}$ II $\mathrm{H}$ contrast and the magnitude of the convective velocity in the photosphere (left), and also between the contrast and the height where convective motions change their sign (middle). In both cases the contrast increases with increasing amplitude of the convective motions and the height where the velocity sign reversal occurs. In our early works, we have shown that not only does intensity contrast reversal occur in granulation, but so to does the reversal of the velocity sign (Kostik \& Khomenko 2012). The plasma was shown to overshoot to higher heights at locations with stronger convective flows in the bottom photosphere, and also at locations with larger magnetic field. The right panel of Fig. 4 completes this picture, demonstrating that, at locations of higher convective velocities and higher overshooting height, the Ca II $\mathrm{H}$ contrast of a plage region also increases.

Figure 5 demonstrates how the wave motions influence the Ca II H contrast. The left upper panel (indicated as "a") reveals that the contrast gets larger at locations where the phase shift $\phi(V, V)$ between velocities at the bottom and upper photosphere (as determined from Ba II profiles) is negative. According to our notation, a negative velocity phase shift means that waves propagate upwards. The phase shift is measured independently at each spatial point, at a frequency with a maximum crosscorrelation coefficient between oscillations at both heights. In all cases, this frequency lies in the five-minute band, with periods that vary between 270 and $330 \mathrm{~s}$. These variations in periods do not influence the results presented in Fig. 5 since there is only a weak dependence between the period and the $\phi(V, V)$ shift. This dependence is such that waves with larger periods have slightly larger negative $\phi(V, V)$ shift, while for waves with smaller periods the $\phi(V, V)$ shift turns positive. The $3 \mathrm{mHz}$ waves observed in the photosphere are essentially evanescent, therefore their velocity phase shift with height is expected to be close to zero from theoretical considerations. Small non-zero phase shifts can be due to 5-min wave propagation enabled either by the ramp effect of inclined magnetic field lines, or by radiative losses. Here, we find that at locations with waves traveling upwards (i.e. larger negative velocity phase shifts) the brightness of the facula gets larger. The brightness increases with increasing upward propagation speed (larger negative values of the velocity phase shift).

The dependence of the Ca II $\mathrm{H}$ contrast on the temperaturevelocity phase shift, $\phi(T, V)$, of upper photospheric oscillations measured from the line core variations of Ba II has a more complex shape (upper middle panel in Fig. 5 , indicatd as "b"). To get the temperature-velocity phase shift we measured the velocityintensity phase shift for oscillations with maximum power (periods in the range 270-330 s in the photosphere) and assumed that temperature and intensity oscillations are $180 \mathrm{deg}$ out of phase. This conversion was done because we deal with a line of a singly ionized element. An increase in temperature leads to an increase in the number of atoms in the ionized state absorbing the radiation. The line becomes deeper and its intensity decreases; i.e., the temperature and intensity oscillations are $180 \mathrm{deg}$ out of phase, see also Shchukina et al. (2009). Similar assumption was done in Kostik \& Khomenko (2013). We operate in terms of temperature-velocity phase shift for a better comparison with theoretical models, see Noyes \& Leighton (1963), Mihalas \& Toomre (1981, 1982), Deubner (1990). The facular contrast is minimum for phase-shift values around $\pm 180^{\circ}$ and has a broad maximum between $0^{\circ}$ and $100^{\circ}$.

The right upper panel of Fig. 5 completes the picture of the contrast dependence on the wave phase shifts by showing a bidimensional dependence. This reveals that not all combinations of the $\phi(T, V)$ and $\phi(V, V)$ phase shifts are present in our data. Locations with enhanced Ca II $\mathrm{H}$ contrast correspond to the locations where two conditions are simultaneously satisfied: the $\phi(V, V)$ phase shift is essentially negative, i.e., waves are propagating upwards, and the $\phi(T, V)$ phase shift is comprised of between $0^{\circ}$ and $120^{\circ}$. These values of the temperature-velocity phase shift are characteristic for acoustic-gravity waves that are strongly affected by radiative losses, and have been frequently detected in earlier observations, see Noyes \& Leighton (1963), Deubner \& Fleck (1989), Fleck \& Deubner (1989). Other combinations of the $\phi(T, V)$ and $\phi(V, V)$ phase shifts are also present. For positive values of $\phi(V, V)$, i.e., downward wave propagation, the $\phi(T, V)$ shifts are essentially either negative or above $120^{\circ}$. In these cases, the $\mathrm{Ca}$ II $\mathrm{H}$ contrast is at its lowest values.

The lower panels of Fig. 5 show the dependence between the power of velocity and intensity oscillations in the upper photosphere, and the Ca II H contrast. There is a weak evidence that the contrast grows with increasing velocity oscillation power, while it is independent of the intensity oscillation power. The 

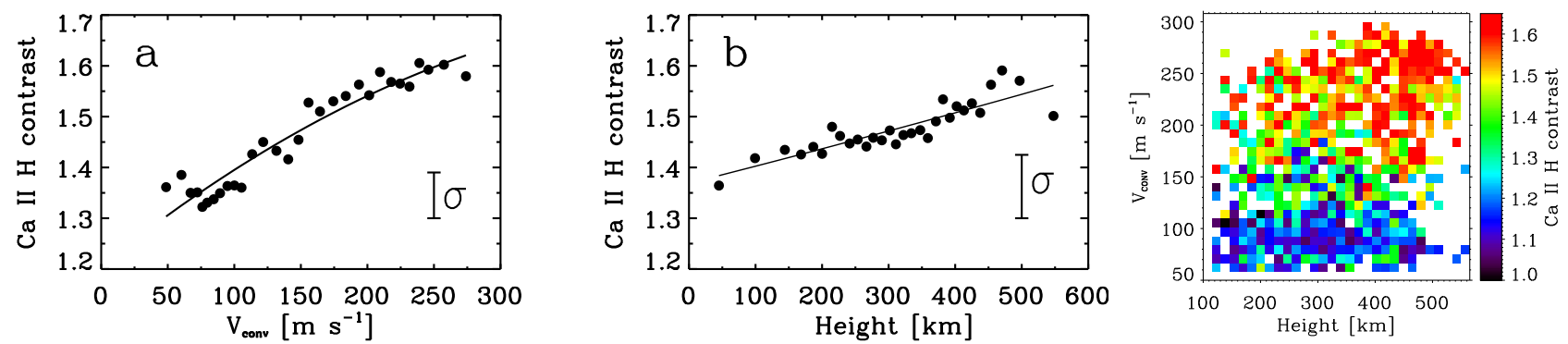

Fig. 4. Ca II $\mathrm{H}$ contrast as a function of the parameters of convection. Left panel: contrast as a function of the amplitude of convective motions at the bottom photosphere. Middle panel: contrast as a function of height where convective velocities change their sign. Each point represents an average value over a bin that contains an equal number of data points on the abscissa axis. The uncertainty $\sigma$ is measured as a standard deviation between the average and the individual values for each bin and then averaged for all bins. Right panel: bi-dimensional representation of the Ca II $\mathrm{H}$ contrast as a function of parameter of convection. Each color rectangle in the scatter plot indicates the mean value of the contrast for a given range of convective velocity and height of sign reversal. The white areas are the locations where a particular combination of convective velocities and reversal heights is not present in our data.
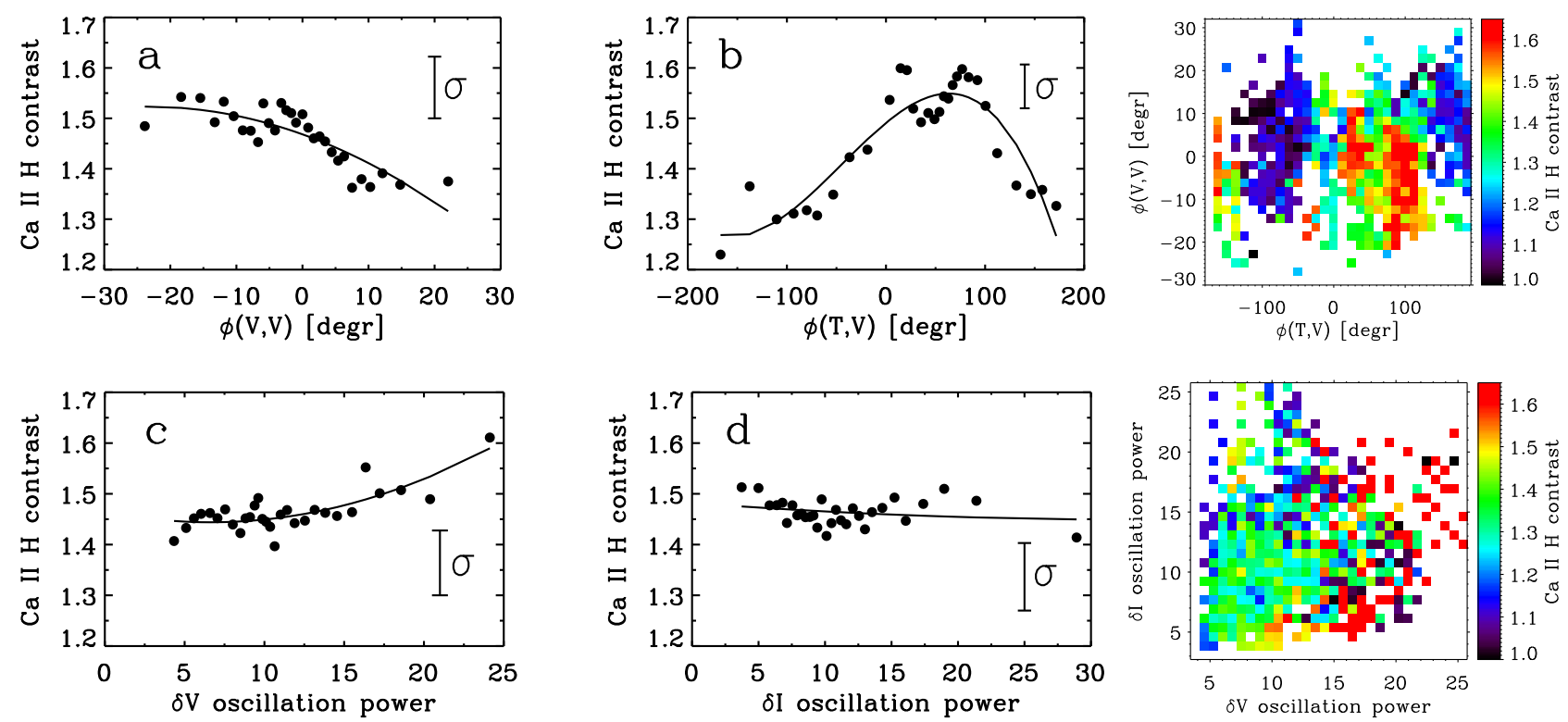

Fig. 5. Ca II H contrast as a function of the parameters of wave motions. Upper left panel: contrast as a function of the phase shift between velocity oscillations at the bottom and upper photosphere for a period with maximum power, $\phi(V, V)$. Upper middle panel: contrast as a function of the phase shift between the temperature and velocity oscillations at the base of the photosphere, $\phi(T, V)$. Upper right panel: bi-dimensional representation of the Ca II $\mathrm{H}$ contrast as a function of $\phi(V, V)$ and $\phi(T, V)$. Bottom left panel: contrast as a function of the power of velocity oscillations. Bottom middle panel: contrast as a function of intensity oscillations, in arbitrary units. Bottom right panel: bi-dimensional representation of the $\mathrm{Ca}$ II $\mathrm{H}$ contrast as a function of velocity and intensity oscillation power.

bi-dimensional representation of the same dependencies that are provided in the lower right-hand panel confirms this conclusion: larger Ca II H contrast is observed in the chromsphere at locations where the velocity oscillations are stronger, but there is a large scatter for the intensity oscillations. Similar conclusions can be also derived from the visual comparison of the spatial distribution of the velocity oscillation power and the power of $\mathrm{Ca}$ II H intensity oscillations from the middle panels of Fig. 2. The patches with enhanced intensity oscillations are smaller and more localized and the dependence between the contrast the intensity oscillation power is not apparent.

\section{Discussion}

The dependences between the chromospheric facular brightness and the parameters of oscillations (Fig. 5) are in agreement with an intuitive picture of upward running waves transferring their energy to the chromosphere and producing an increase in its brightness. Indeed, according to analysis of the same dataset in (Kostik \& Khomenko 2013), in about $67 \%$ of locations in the observed area, the $\phi(V, V)$ is negative, i.e., in most of the area the waves are propagating upwards. The greater the power of velocity oscillations is, the larger the facular contrast (Fig. 5c). Intensity oscillations affect the facular contrast less, possibly because of the magnetic nature of the observed waves, in which case the principal restoring force is the magnetic field and oscillations of thermodynamic parameters are less important.

Figure 6 provides additional details on the relation between the wave propagation direction, their power, and $\mathrm{Ca}$ II $\mathrm{H}$ contrast. The left and middle panels give similar dependencies as panel $\mathrm{c}$ of Fig. 5, but this time separating into upward and downward running waves. We note that the contrast increases with the wave power only for upward running waves (left panel). For the downward running ones (right panel), the dependence is just the opposite. A similar conclusion is also apparent from the bi-dimensional representation of the contrast as a function of the 

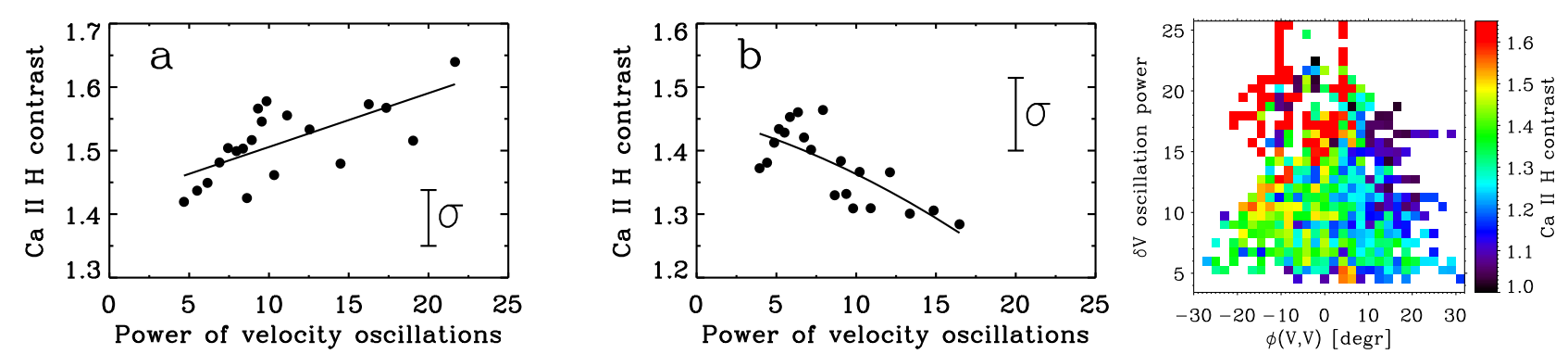

Fig. 6. Left panel: Ca II H contrast as a function of power of upward propagating waves. Middle panel: contrast as a function of the power of downward propagating waves. Right panel: bi-dimensional representation of the Ca II $\mathrm{H}$ contrast as a function of velocity phase shift, $\phi(V, V)$, indicative of the direction of the wave propagation, and velocity oscillation power.
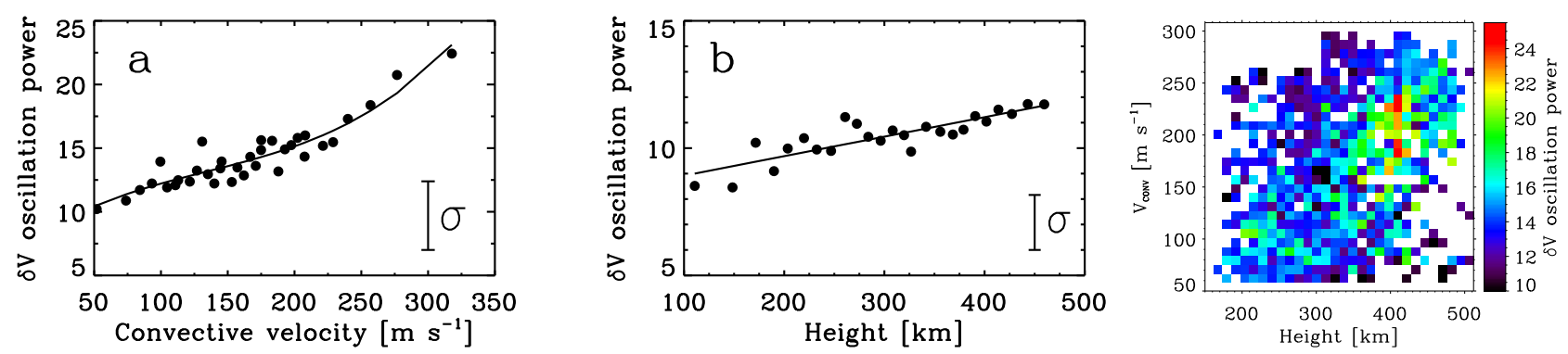

Fig. 7. Left panel: power of velocity oscillations in the Ba II line core as a function of the amplitude of convective motions at the photospheric base. Middle panel: same quantity as a function of height of sign reversal of convective motions. Right panel: bi-dimensional representation of the velocity oscillation power as a function of the strength of convective motions and their reversal height.

velocity oscillation power and $\phi(V, V)$, see right panel of Fig. 6. The contrast is maximum where two conditions are simultaneously satisfied: waves are propagating upwards and their power is maximum. This result needs further study.

As was shown in our previous work (Kostik \& Khomenko 2013), the 5-min oscillations more effectively reach the temperature minimum from the bottom photosphere when the phase shift between the oscillations of temperature and velocity are in the range of $0^{\circ}$ and $90^{\circ}$. If these waves were observed in a quiet area, this type of phase shift would suggest clear deviations from adiabatic wave propagation (an adiabatic propagation leads to $180^{\circ}$ phase shift). While $\phi(V, V)$ phase shifts provide for a more straightforward interpretation, in terms of the direction of the wave propagation, it is not so for the $\phi(T, V)$ phase shifts (Deubner 1990). Solar 5-min oscillations are strongly affected by radiative losses and the precise value of the $\phi(T, V)$ phase shift depends on the amount of radiative losses, wave frequency, and the height in the atmosphere where the waves are observed (see Mihalas \& Toomre 1981, 1982; Deubner 1990; Deubner \& Fleck 1989; Fleck \& Deubner 1989, among others). Owing to the rapid change in height of the sound speed (temperature), wave reflection can also occur and standing waves can be produced. In this case, the values of the $\phi(T, V)$ phase shift of $90^{\circ}$ would also appear if the wave propagation was considered adiabatic. However, there are arguments against this kind of interpretation. On the one hand, there have been theoretical studies of the acoustic-gravity wave reflection (e.g., Marmolino et al. 1993) which showed that the reflection coefficient is small in the evanescent region of the $k-\omega$ diagram between the Lamb frequency and the acoustic cut-off frequency (i.e. in the range of frequencies similar to that in our data). On the other hand, standing waves can be discarded in the upper right panel of Fig. 5 since these show negative values of the $\phi(V, V)$ phase shifts (upward propagation) when $\phi(T, V)$ phase shifts lie in the range between $0^{\circ}$ and $90^{\circ}$. Extra care, however, has to be take when interpreting
$\phi(T, V)$ phase shifts in our case, since the waves are observed in a strongly magnetized facular area and the dependence between the temperature-velocity phase shift and non-adiabaticity of oscillations in the presence of the magnetic field is not well studied theoretically (see the discussion in Kostik \& Khomenko 2013). Determining the phase shift between temperature and velocity for magneto-acoustic waves would need numerical modeling for particular cases of the observed magnetic field geometries.

It is intriguing that the facular contrast in the chromosphere depends on the strength of convective motions in the photosphere, given that flows do not reach chromospheric heights. Kostyk et al. (2006) find that the power of 5-min oscillations at the formation height of the Fe I $6393.6 \AA$ spectral line (around $500 \mathrm{~km}$ ) increases with increasing strength of convective motions at the bottom photosphere. It is then possible that something similar happens for the Ba II line. Figure 7 shows the dependence between the power of 5-min oscillations at the core of Ba II line and the convective velocity, demonstrating that the wave power increases with increasing strength of convective motions, similar to findings in Kostyk et al. (2006). The wave power also increases with increasing height where the convective motions change their sign (middle panel of Fig. 7), and is maximum at locations where both conditions (large convective velocity and higher reversal height) are satisfied, see right panels of Fig. 7. Therefore, it can be concluded that convective motions, by exciting oscillations, indirectly contribute to the increase of facular brightness in the chromosphere.

\section{Conclusions}

Due to the presence of the magnetic field in the facular area, 5-min waves penetrate to chromospheric heights (either along the magnetic field lines or owing to non-adiabatic effects), and lead to an efficient energy transfer to the chromosphere of the solar facula. The brightness of the facula was found to strongly 
depend on the power of waves traveling upwards. The convective motions at the photospheric base influence the brightness in an indirect way. The larger the amplitude of convective motions is and the height in the photosphere where they change sign, the brighter the facula becomes. Together, all these results lead to the conclusion that facular areas appear bright not only because of the Wilson depression, but also because of real heating.

Acknowledgements. This work is partially supported by the Spanish Ministry of Science through projects AYA2010-18029, AYA2011-24808, and AYA2014 55078-P. This work contributes to the deliverables identified in FP7 European Research Council grant agreement 277829 "Magnetic connectivity through the Solar Partially Ionized Atmosphere".

\section{References}

Berger, T. E., Rouppe van der Voort, L. H. M., Löfdahl, M. G., et al. 2004, A\&A 428,613

Collados, M., Lagg, A., Díaz Garcí A, J. J., et al. 2007, in The Physics of Chromospheric Plasmas, eds. P. Heinzel, I. Dorotovič, \& R. J. Rutten, ASP Conf Ser., 368, 611

Deubner, F. L. 1990, in The Solar Photosphere: Structure, Convection and Mag netic Fields, ed. J.-O. Stenflo, Proc. IAU Symp. 138 (Kiev) (Dordrecht Kluwer), 217

Deubner, F.-L., \& Fleck, B. 1989, A\&A, 213, 423

Dunn, R. B., \& Zirker, J. B. 1973, Sol. Phys., 33, 281

Fleck, B., \& Deubner, F.-L. 1989, A\&A, 224, 245

Frazier, E. N. 1971, Sol. Phys., 21, 42

Grossmann-Doerth, U., Knoelker, M., Schuessler, M., \& Solanki, S. K. 1994 A\&A, 285, 648
Khomenko, E. V., Kostik, R. I., \& Shchukina, N. G. 2001, A\&A, 369, 660 Knoelker, M., Schuessler, M., \& Weisshaar, E. 1988, A\&A, 194, 257 Kobel, P., Solanki, S. K., \& Borrero, J. M. 2011, A\&A, 531, A112 Kostik, R., \& Khomenko, E. V. 2012, A\&A, 545, A22

Kostik, R., \& Khomenko, E. 2013, A\&A, 559, A107

Kostik, R., Khomenko, E., \& Shchukina, N. 2009, A\&A, 506, 1405 Kostyk, R. I. 2013, Kinematics and Physics of Celestial Bodies, 29, 32

Kostyk, R. I., Shchukina, N. G., \& Khomenko, E. V. 2006, Astron. Rep., 50, 588

Lites, B. W., Scharmer, G. B., Berger, T. E., \& Title, A. M. 2004, Sol. Phys., 221, 65

Marmolino, C., Severino, G., Deubner, F.-L., \& Fleck, B. 1993, A\&A, 278, 617

Ruiz Cobo, B., \& del Toro Iniesta, J. C. 1992, ApJ, 398, 375

Mihalas, B. W., \& Toomre, J. 1981, ApJ, 249, 349

Mihalas, B. W., \& Toomre, J. 1982, ApJ, 263, 386

Montagne, M., Mueller, R., \& Vigneau, J. 1996, A\&A, 311, 304

Narayan, G., \& Scharmer, G. B. 2010, A\&A, 524, A3

Noyes, R. W., \& Leighton, R. W. 1963, ApJ, 138, 631

Okunev, O. V., \& Kneer, F. 2005, A\&A, 439, 323

Shchukina, N. G., Olshevsky, V. L., \& Khomenko, E. V. 2009, A\&A, 506, 1393

Solanki, S. K. 1993, Space Sci. Rev., 63, 1

Spruit, H. C. 1976, Sol. Phys., 50, 269

Stebbins, R. T., \& Goode, P. R. 1987, Sol. Phys., 110, 237

Steiner, O. 2005, A\&A, 430, 691

Stenflo, J. O. 1973, Sol. Phys., 32, 41

Title, A. M., Topka, K. P., Tarbell, T. D., et al. 1992, ApJ, 393, 782

Topka, K. P., Tarbell, T. D., \& Title, A. M. 1992, ApJ, 396, 351

Topka, K. P., Tarbell, T. D., \& Title, A. M. 1997, ApJ, 484, 479

Tritschler, A., Schmidt, W., Langhans, K., \& Kentischer, T. 2002, Sol. Phys., 211, 17

Viticchié, B., Sánchez Almeida, J., Del Moro, D., \& Berrilli, F. 2011, A\&A, 526, A60 